\title{
MS and COVID-19 challenge: asymptomatic COVID-19 infection during treatment with cladribine
}

\author{
Meral Seferoğlu' ${ }^{1}(0)$ Özlem Ethemoğlu ${ }^{2}$ Ömer Faruk Turan ${ }^{3} \cdot$ Aksel Siva $^{4}$
}

Received: 13 March 2021 / Accepted: 14 June 2021 / Published online: 24 June 2021

(c) Fondazione Società Italiana di Neurologia 2021

\begin{abstract}
Background The use of disease-modifying therapies (DMTs) in people with multiple sclerosis (pwMS) may affect COVID19 infection outcomes due to DMTs' immunomodulatory and immunosuppressive effects on immune response. The yet unknown issues are both the early response to the infection, as well as the post-infection development of immunity against the virus under these treatments due to their interaction with the immune system.

Methods We report two asymptomatic cases of COVID-19 in patients with relapsing-remitting multiple sclerosis (RRMS) shortly after starting cladribine therapy, both developed anti-SARS-CoV-2 antibody response.

Results Patients with MS who are under newly initiated treatment with cladribine tablets may experience an asymptomatic COVID-19 infection and they may develop immunity against SARS-CoV-2.

Conclusion These observations raise the probability that DMTs with immunosuppressive effects, such as cladribine, may be considered as a treatment option for selected MS patients with high disease activity during the COVID-19 pandemic.
\end{abstract}

Keywords Multiple sclerosis · Cladribine $\cdot$ COVID-19 $\cdot$ SARS-CoV-2 antibody response

\section{Background}

The accumulated information since the beginning of the pandemic has confirmed that people with multiple sclerosis (pwMS) have a similar risk and experience the COVID-19 infection as the non-MS population, with advanced age, obesity, and several comorbid conditions increasing the risk for having a more severe course, as well as disease-specific features such as having a progressive course, increased

Meral Seferoğlu

meralbozseferoglu@gmail.com

Aksel Siva

akselsiva@gmail.com

1 Department of Neurology, Bursa Yüksek İhtisas Training and Research Hospital, University of Health Sciences, Bursa, Turkey

2 Department of Neurology, Harran University School of Medicine, Sanliurfa, Turkey

3 Department of Neurology, Uludag University School of Medicine, Bursa, Turkey

4 Department of Neurology and MS Clinic, Cerrahpasa School of Medicine, Istanbul University-Cerrahpasa, Istanbul, Turkey disability, and longer disease duration [1]. None of the MS treatments had been shown to increase the risk for a more severe COVID-19 infection with the exception of B-cell depleting treatments [2]. However, since the outbreak of pandemic, most of the MS Societies had advised to delay or not to initiate disease-modifying therapies (DMTs) with a more potent immunosuppressive effect such as ocrelizumab, rituximab, alemtuzumab, and cladribine [3].

Here, we report our experience with two MS patients who developed asymptomatic COVID-19 infection soon after DMT was initiated with cladribine.

\section{Case reports}

\section{Case 1}

A 29-year-old female was diagnosed with relapsing-remitting MS in 2013 and glatiramer acetate was initiated. On August 2020, she had an acute MS attack consisting of diplopia, numbness and weakness on the right side, and ataxia. Her MRI had disclosed multiple enhancing cranial and spinal lesions. She was treated with pulse intravenous methylprednisolone and improved to her baseline status of 
EDSS2. Due to increased disease activity and lack of comorbid conditions at a time when the pandemic had declined in Turkey, a shared treatment decision to switch to cladribine was made. Of the two treatment-week cycles of the first year, she had received the initial one starting on 30 September and the second one on 30 October 2020 . On the 4th day of the second course, patient's husband complained about headache and fatigue and his diagnosis of COVID-19 infection was confirmed. She was also tested and was found positive for SARS-CoV-2-RNA on naso-oropharyngeal swabs. She did not develop any symptoms of COVID-19 infection and her thoracic tomography was normal. Her immunological profile was evaluated and is shown in Table 1. After 23 days of her initial positive RT-PCR test, she tested positive for anti-SARS-CoV-2-IgG antibodies on CMIA (Table 1).

\section{Case 2}

A 45-year-old female patient who was diagnosed with relapsing-remitting multiple sclerosis in March 2014 was treated earlier with interferon beta-1a, teriflunomide, and fingolimod, but due to continuous high disease activity was decided to be switched to cladribine treatment on August 31, 2020. Her EDSS was 2.5. She received the first week cycle of cladribine on 31 August and the second cycle on 1 October 2020. On 9 November, following her son's diagnosis of COVID-19 infection, she was tested positive on nasooropharyngeal swabs for SARS-CoV-2-RNA. However, she remained asymptomatic, and a follow-up test was negative 3 weeks later. Her anti-SARS-CoV-2-IgG test done at the same time was negative, but when repeated on sixth week of her initial positive swab it became positive for anti-SARSCoV-2-IgG (Table 1).

\section{Discussion}

Initially, with the onset of the COVID-19 pandemic, treatment options with immunosuppressive DMTs in pwMS became limited due to the probability that they may increase the risk of the infection and developing complications. However, after a number of studies had shown that this was not the case $[1,2]$, the recent tendency became that such treatments should be continued or even may be initiated in pwMS who have high disease activity after individual benefit-risk evaluations [4].

Cladribine is a DMT that has a long-term efficacy due to its mode of action. It shows a selectivity towards proinflammatory immune cell subsets, resulting in transient depletion of memory B cell and TH17 cell populations, while leaving the innate immune system intact [5]. The modest depletion induced by cladribine in $\mathrm{T}$ and NK cells and a more marked depletion of $\mathrm{B}$ cells are seen most significantly between the first and third months [5].

As of 29 June 2020, approximately there were 19,000 pwMS who have been treated with cladribine and 18 of them had confirmed COVID-19 infection within the safety database [6]. The majority of patients with suspected or confirmed COVID-19 infection had mild to moderate respiratory symptoms with two of them remaining asymptomatic [6].

Cladribine has received reimbursement for MS treatment in Turkey on May 2020 and was given to 230 MS
Table 1 Panel showing laboratory data of two cases after COVID-19 infection

\begin{tabular}{llll}
\hline Tests* & Case 1 & Case 2 & Reference range \\
\hline White blood cells & 3890 & 3600 & $3500-10,500(\mu \mathrm{L})$ \\
Lymphocytes & 1120 & 940 & $1090-2900(\mu \mathrm{L})$ \\
Neutrophil & 2530 & 1990 & $1800-7000(\mu \mathrm{L})$ \\
CD4/CD8 & 1.59 & 0.94 & $0.8-3.9$ \\
CD19 & 0.70 & 3.10 & $7-23(\%)$ \\
CD20 & 0.70 & 3.20 & $6-23(\%)$ \\
IgA & 1.98 & 2.01 & $0.46-2.21(\mathrm{~g} / \mathrm{L})$ \\
IgM & 2.17 & 0.644 & $0.45-1.98(\mathrm{~g} / \mathrm{L})$ \\
IgG & 15 & 6.29 & $7.3-16.20(\mathrm{~g} / \mathrm{L})$ \\
IL-6 & 5.98 & NA & $0-3.4(\mathrm{pg} / \mathrm{mL})$ \\
Anti-SARS-CoV-2-IgG** & 3.19 (3rd week) & Negative (3rd week) & $<1.4$ \\
& & Positive (9th week) & \\
\hline
\end{tabular}

*These data were obtained on the same day the last cladribine tablet was given and after the RT-PCR swab test came positive for COVID-19 infection for case 1 and on the 35th day for case 2

** For case 1, the anti-SARS-CoV-2-IgG test used was chemiluminescent microparticle immunoassay (CMIA) from Abbott Diagnostics which is a 2-step qualitative assay detecting in serum or plasma IgG antibodies against the SARS-CoV-2 nucleocapsid protein, performed on an Architect i2000. For case 2, the test used was ELISA based with a positive value being over 6 
patients since then. These two patients are currently the only cladribine-treated patients from this cohort who became positive on RT-PCR-test for COVID-19 infection. Their immunoglobulins and CD4/CD8 ratio were within normal limits; CD19 and CD20-B-cell to lymphocyte ratios were low in both. First case had elevated levels of IL-6 and second case had grade I lymphopenia (Table 1). Interestingly, both remained asymptomatic with their follow-up RT-PCR test becoming negative within 2 weeks and both developed anti-SARS-CoV-2-IgG.

It was suggested that asymptomatic individuals had a weaker immune response to SARS-CoV-2 infection [7] and it is probable that cladribine may have contributed to this response in our patients as it is also known that this treatment decreases the levels of proinflammatory cytokines [8]. Data from randomized-controlled trials suggest that infections with cladribine are infrequent and self-limiting despite peripheral lymphocyte depletion [9]. It is not clear whether lymphopenia is a risk factor for a more severe COVID-19 infection or on the contrary that it may be beneficial by preventing the severe inflammatory response [10].

Severity of COVID-19 infection in patients with MS is closely related to either comorbidities or disease-specific factors rather than MS treatments with the probable exception of B-cell depleting therapies. Since MS may pose a more serious risk than COVID-19 infection in the long run, the most appropriate treatment should be considered after a proper risk-benefit analysis.

In conclusion, our experience illustrates that even during active treatment with cladribine the patients may acquire COVID-19-infection with no symptoms and develop antiSARS-CoV-2 antibodies. While there is no clear-cut evidence for the effects of cladribine on COVID-19, our cases suggest that cladribine may not increase the risk of COVID19 severity in pwMS without comorbidity and may be considered as a treatment option for selected MS patients with high disease activity. Besides, the overall observations of asymptomatic or mild COVID-19 in pwMS under DMTs rise some further questions like whether these treatments are somehow related to prevent a more severe infection risk or that the immunity of MS itself has something to do with such a favorable outcome in COVID-19 patients.

\section{Declarations}

Ethical approval Written informed consent was obtained from the patients to report their individual cases.
Conflict of interest The authors declare no competing interests.

\section{References}

1. Sormani MP, De Rossi N, Schiavetti I, et al (2021) Disease modifying therapies and Covid-19 severity in multiple sclerosis. Ann Neurol 2021/01/23. https://doi.org/10.1002/ana.26028

2. Berger JR, Brandstadter R and Bar-Or A (2020) COVID-19 and MS disease-modifying therapies. Neurol Neuroimmunol Neuroinflamm; 7 2020/05/18. https://doi.org/10.1212/NXI.0000000000 000761

3. https://www.msif.org/ (accessed 26th December 2020)

4. Zrzavy T, Wimmer I, Rommer PS, et al (2020) Immunology of COVID-19 and disease-modifying therapies: the good, the bad and the unknown. Eur J Neurol 2020/10/23. https://doi.org/10. 1111/ene. 14578

5. Moser T, Schwenker K, Seiberl M, et al (2020) Long-term peripheral immune cell profiling reveals further targets of oral cladribine in MS. Ann Clin Transl Neurol; 7: 2199-2212. 2020/10/02. https://doi.org/10.1002/acn3.51206

6. Jack D, Nolting A and Galazka A (2020) Favorable outcomes after COVID-19 infection in multiple sclerosis patients treated with cladribine tablets. Mult Scler Relat Disord; 46: 102469. 2020/09/13. https://doi.org/10.1016/j.msard.2020.102469

7. Long QX, Tang XJ, Shi QL, et al (2020) Clinical and immunological assessment of asymptomatic SARS-CoV-2 infections. Nat Med; 26: 1200-1204. 2020/06/20. https://doi.org/10.1038/ s41591-020-0965-6

8. Laugel B, Borlat F, Galibert L, et al (2011) Cladribine inhibits cytokine secretion by $\mathrm{T}$ cells independently of deoxycytidine kinase activity. J Neuroimmunol; 240-241: 52-57. 2011/11/01. https://doi.org/10.1016/j.jneuroim.2011.09.010

9. Cook S, Leist T, Comi G, et al (2019) Safety of cladribine tablets in the treatment of patients with multiple sclerosis: an integrated analysis. Mult Scler Relat Disord; 29: 157-167. 2019/03/20. https://doi.org/10.1016/j.msard.2018.11.021

10. Parrotta E, Kister I, Charvet L, et al (2020) COVID-19 outcomes in MS: observational study of early experience from NYU Multiple Sclerosis Comprehensive Care Center. Neurol Neuroimmunol Neuroinflamm; 7 2020/07/11. https://doi.org/10.1212/NXI.00000 00000000835

Publisher's note Springer Nature remains neutral with regard to jurisdictional claims in published maps and institutional affiliations. 